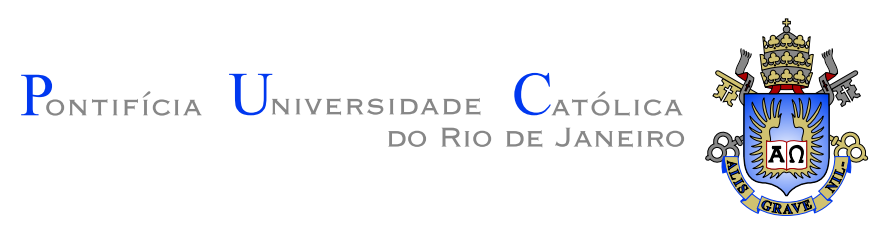

Julio Rául Sierra Vásquez

\title{
Redução de atrito em escoamento laminar por lubrificação de parede com ranhuras
}

\section{Dissertação de Mestrado}

Dissertação apresentada como requisito parcial para obtenção do grau de Mestre pelo Programa de Pós-graduação em Engenharia Mecânica do Departamento de Engenharia Mecânica da PUC-Rio

Orientador : Prof. Márcio da Silveira Carvalho Co-Orientador: Prof. Luís Fernando Alzuguir Azevedo 


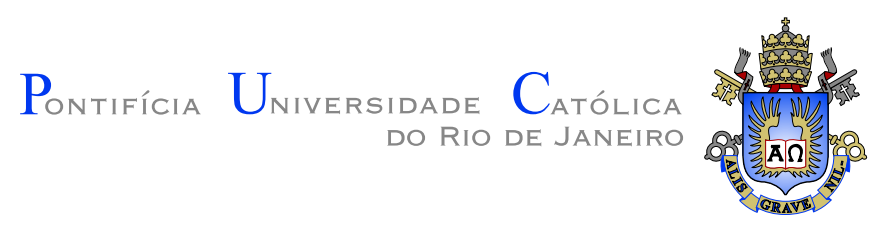

Julio Rául Sierra Vásquez

\title{
Redução de atrito em escoamento laminar por lubrificação de parede com ranhuras
}

Dissertação apresentada como requisito parcial para obtenção do grau de Mestre pelo Programa de Pós-graduação em Engenharia Mecânica do Departamento de Engenharia Mecânica do Centro Técnico Científico da PUC-Rio. Aprovada pela Comissão examinadora abaixo assinada.

\author{
Prof. Márcio da Silveira Carvalho \\ Orientador \\ Departamento de Engenharia Mecânica - PUC-Rio
}

Prof. Luís Fernando Alzuguir Azevedo

Co-Orientador

Departamento de Engenharia Mecânica - PUC-Rio

Dr. Geraldo Spinelli Ribeiro
Petrobrás

Dr. Sidney Stuckenbruck

Departamento de Engenharia Mecânica - PUC-Rio

Prof. José Eugenio Leal

Coordenador Setorial do Centro Técnico Científico - PUC-Rio

Rio de Janeiro, 11 de Agosto de 2009 
Todos os direitos reservados. É proibida a reprodução total ou parcial do trabalho sem autorização da universidade, do autor e do orientador.

\section{Julio Rául Sierra Vásquez}

Graduou-se em Engenharia Mecânica pela UCSM ( Universidad Católica de Santa Maria ) em 2002. Experiência em manutenção de maquinaria pesada, Desenho de Estruturas Metálicas

Ficha Catalográfica

Julio Rául Sierra Vásquez

Redução de atrito em escoamento laminar por lubrificação de parede com ranhuras / Julio Rául Sierra Vásquez; orientador: Márcio da Silveira Carvalho; co-orientador: Luís Fernando Alzuguir Azevedo. - Rio de Janeiro : PUC-Rio, Departamento de Engenharia Mecânica, 2009.

v., 53 f: il. ; $29,7 \mathrm{~cm}$

1. Dissertação (mestrado) - Pontifícia Universidade Católica do Rio de Janeiro, Departamento de Engenharia Mecânica.

Inclui referências bibliográficas.

1. Engenharia Mecânica - Tese. 2. Redução de atrito . 3. Escoamento laminar. 4. Superfícies corrugadas. I. Márcio da Silveira Carvalho. II. Luís Fernando Alzuguir Azevedo. III. Pontifícia Universidade Católica do Rio de Janeiro. Departamento de Engenharia Mecânica. IV. Título. 
Dedico este trabalho a meu pai e a minha família pelo apoio e a meus orientadores pelo ensino dados nestes dois anos. 


\section{Agradecimentos}

A meu pai e irmãos pela confiança em mim e o apoio dado nestes dois anos.

Ao professor Márcio da Silveira Carvalho e ao professor Luis Fernando Alzuguir Azevedo pela orientação neste trabalho.

Ao CNPq pelo apoio na bolsa de estudos.

Aos membros da banca pela participação e sugestões.

A meus amigos e demais pessoas que me apoiaram neste tempo.

Ao pessoal do grupo de REOLOGIA, pela ajuda dada. 


\section{Resumo}

Julio Rául Sierra Vásquez; Márcio da Silveira Carvalho; Luís Fernando Alzuguir Azevedo. Redução de atrito em escoamento laminar por lubrificação de parede com ranhuras. Rio de Janeiro, 2009. 53p. Dissertação de Mestrado — Departamento de Engenharia Mecânica, Pontifícia Universidade Católica do Rio de Janeiro.

Objetivo: Uma parte significativa das reservas mundiais de petróleo é encontrada na forma de óleos pesados. Estes óleos pesados possuem alta viscosidade de 100 - $10000 \mathrm{cP}$, que torna seu transporte altamente complexo e custoso. Vários métodos foram desenvolvidos para reduzir a perda de carga de escoamentos laminares de óleos de alta viscosidade. Entre os mais utilizados, pode-se citar o bombeio de um fluido de baixa viscosidade perto da parede do tubo com o óleo viscoso sendo transportado no centro, conhecido como "core-annular flow". Neste trabalho, uma alternativa ao core-annular flow é estudada. O método é baseado na utilização de micro ranhuras da parede do duto preenchidas com um liquido de baixa viscosidade. Este método tem o potencial de evitar alguns dos problemas que ocorrem no uso de core-annular flow. A análise do efeito das diferentes propriedades dos fluidos, condições de operação, geometria das ranhuras na perda de carga do escoamento foi feita através de um estudo numérico e experimental. Resultados indicam as limitações e potencialidade do uso de micro ranhuras na redução de perda de carga de escoamento laminar

\section{Palavras-chave}

Redução de atrito . Escoamento laminar. Superfícies corrugadas. 


\section{Abstract}

Julio Rául Sierra Vásquez; Márcio da Silveira Carvalho; Luís Fernando Alzuguir Azevedo. Drag reduction in laminar flow by lubrication of grooved walls. Rio de Janeiro, 2009. 53p. MsC Dissertation Departament of Engenharia Mecânica, Pontifícia Universidade Católica do Rio de Janeiro.

Objective: A significant portion of the world oil reserves is found in the form of heavy oil. These oils have a high values of viscosity around 100-10000 cP, that makes their transportation complex and expensive. Several methods have been developed to reduce the pressure drop in laminar flows of high viscosity oils. Among them is the solution of pumping a liquid of lower viscosity near the pipe wall with the high viscosity oil flowing in the center. This method is known as Core-annular flow. In this work, an alternative to core-annular flow is studied. The method is based on the use of micro grooves in the pipe wall filled with a liquid of smaller viscosity. This method has the potential to elude some problems that occur with the core-annular flow method. The analysis of the drag reduction effect as a function of different fluids properties, operational conditions and geometry of the grooved walls was made using a numerical and experimental approach. Results indicate the limitations and potential of using micro grooves for drag reduction in laminar flows of high viscosity fluids.

\section{Keywords}

Drag reduction. Laminar Flow. Grooved walls. 


\section{Sumário}

$\begin{array}{ll}\text { Sumário das notações } & 13\end{array}$

1 Introdução $\quad 14$

1.1 Motivação 14

$\begin{array}{lll}1.2 & \text { Core-annular flow (CAF) } & 15\end{array}$

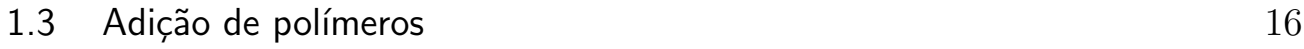

$\begin{array}{lll}1.4 & \text { Micro ranhuras } & 17\end{array}$

2 Análise numérica $\quad 19$

2.1 Equações de conservação 19

2.2 Solução do escoamento entre placas planas. 19

2.3 Solução do problema com parede deslizantes 20

2.3.1 Resultados 21

2.4 Solução do problema com interface entre o líquido viscoso e o líquido lubrificante nas ranhuras.

2.4.1 Condições de contorno da equação de conservação de quantidade de movimento.

2.4.2 Condições de contorno de malha 24

2.5 Solução computacional 26

2.5.1 Configuração da malha 26

2.5.2 Resultados da simulação 29

2.5.3 Fator de atrito para placas planas lisas e com ranhuras 35

3 Análise experimental $\quad 38$

3.1 Dispositivo experimental 38

3.2 Preparação da bancada 41

3.3 Procedimento experimental $\quad 42$

3.4 Resultados Experimentais 43

3.5 Fator de atrito em placas planas e com ranhuras. 46

3.6 Comparação da simulação numérica com dados experimentais 48

3.7 Teste de longa duração 49

3.8 Teste de reinicio do escoamento após parada 50

4 Conclusões e comentários finais $\quad 51$

Referências Bibliográficas $\quad 53$ 


\section{Lista de figuras}

$\begin{array}{lll}1.1 & \text { Core Annular Flow Process } & 15\end{array}$

1.2 Estabilidade bifásica num escoamento CAF [? ] 16

$\begin{array}{ll}1.3 \text { Micro Canais } & 17\end{array}$

1.4 Ângulo de contato de uma superfície plana e uma com Nanogrelhas $\begin{array}{ll}\text { [?] } & 18\end{array}$

2.1 Condição de contorno como uma parede deslizante 20

2.2 Problema com superfícies deslizantes 21

2.3 Relação de vazão com o comprimento da parede deslizante 22

2.4 Distribuição de pressão ao longo do comprimento 22

2.5 Queda de pressão ao longo do comprimento 22

2.6 Linhas de corrente ao longo do comprimento 23

2.7 Problema com interface 24

2.8 Condições de momentum 24

2.9 Condição de contorno de malha 25

2.10 Geometria da malha 26

2.11 Malha com uma superfície corrugada de $1 \mathrm{~mm} \quad 27$

2.12 Malha com uma superfície corrugada de $\mathrm{H}=10 \mathrm{~mm} \quad 28$

2.13 Malha com uma superfície corrugada de $H$ e $h$ variável 29

2.14 Contornos de pressão e linhas de corrente numa superfície corrugada de $1 \mathrm{~mm}$ e uma relação de viscosiades $\mu 1 / \mu 2=1$

2.15 Contornos de pressão e linhas de corrente numa superfície corrugada de $2 \mathrm{~mm}$ e uma relação de viscosidades $\mu 1 / \mu 2=1$

2.16 Relação da vazão com a razão da viscosidade para uma superfície corrugada de $L_{0}=1 \mathrm{~mm}, L=3 \mathrm{~mm}, h=1 \mathrm{~mm}, \Delta \mathrm{P}=10 \mathrm{~Pa}, H=5 \mathrm{~mm} .31$

2.17 Relação de vazão $\mathrm{Q} / Q_{0}$ com o comprimento da parede $L_{0} / L$ para uma superfície corrugada de $H=5 \mathrm{~mm}$, com diversas razões de viscosidades

2.18 Relação de vazão com razão de viscosidades para uma superfície corrugada de $L_{0}=1 \mathrm{~mm}, L=3 \mathrm{~mm}, h=1 \mathrm{~mm}, \Delta \mathrm{P}=10 \mathrm{~Pa}, H=10$ $\mathrm{mm}$ e uma relação de viscosidades $\mu 1 / \mu 2=1$

2.19 Relação de vazão $\mathrm{Q} / Q_{0}$ com o comprimento da parede $L_{0} / \mathrm{L}$ para uma superfície corrugada de $\mathrm{H}=10 \mathrm{~mm}$

2.20 Ganho na vazão numa superfície corrugada de $L_{0}=1 \mathrm{~mm}, L=3$ $\mathrm{mm}, h=$ variável, $\Delta \mathrm{P}=10 \mathrm{~Pa}, H=$ variável, em função da razão de viscosidades

2.21 Fator de atrito modulado pelo número de Reynolds para uma superfície corrugada de $L_{0}=$ variável, $L=3 \mathrm{~mm}, h=1 \mathrm{~mm}, \Delta \mathrm{P}=10$ $\mathrm{Pa}, H=5 \mathrm{~mm}$ e $\mu 1 / \mu 2$ de 1,10 e 100

2.22 Fator de atrito modulado pelo número de Reynolds para uma superfície corrugada de $L_{0}=$ variável, $L=3 \mathrm{~mm}, \mathrm{~h}=1 \mathrm{~mm}, \Delta \mathrm{P}=10$ $\mathrm{Pa}, H=10 \mathrm{~mm}$ e $\mu 1 / \mu 2$ de 1,10 e 100

3.1 Representação gráfica da bancada experimental 
3.2 Dispositivo de acrílico construído para obter um escoamento entre duas placas paralelas

3.3 Placas ranhuras de $1 \mathrm{~mm} \times 1 \mathrm{~mm} \quad 40$

3.4 Placas ranhuras de $0,3 \mathrm{~mm} \times 1 \mathrm{~mm}$

3.5 Placas Testadas no Experimento 41

3.6 Bancada experimental 42

3.7 Vista das Placas com ranhuras montadas 43

3.8 Queda de pressão para uma placa plana lisa, teórico e experimental. 44

3.9 Queda de pressão em função da vazão entre duas placas planas com ranhuras de $1 \mathrm{~mm}$ de largura, enchidas com um fluido lubrificante. 44

3.10 Interface entre um fluido altamente viscoso e o fluido lubrificante com ranhuras de $1 \mathrm{~mm}$.

3.11 Queda de pressão em função da vazão imposta, para glicerina escoando entre duas placas planas e placas com ranhuras de 0,3 $\mathrm{mm}$ preenchidas com um fluido lubrificante

3.12 Linha de interface entre um liquido de alta viscosidade e o liquido lubrificante

3.13 Comparação de $f \times R e$ para placas planas como o valor teórico 48

3.14 Comparação de $f \times R e$ para placas com ranhuras com o valor teórico para placa plana

3.15 Comparação experimental e numérica para uma configuração de comprimento de ranhura de $L o=0,3 \mathrm{~mm}$. 


\section{Lista de tabelas}

2.1 Resultados preliminares da simulação (Placas deslizantes) 21

2.2 Superfície corrugada de $L_{0}$ variável, $L=3 \mathrm{~mm}, h=1 \mathrm{~mm}, \Delta \mathrm{P}=10$ $\mathrm{Pa}$.

2.3 Superfície corrugada de $L_{0}$ variável, $L=3 \mathrm{~mm}, h=1 \mathrm{~mm}, \Delta \mathrm{P}=10$ $\mathrm{Pa}$.

2.4 Superfície corrugada de $L_{0}=1 \mathrm{~mm}, L=3 \mathrm{~mm}, h=$ variável, $\Delta \mathrm{P}=10$ $\mathrm{Pa}, H=$ variável.

2.5 Geometria para uma superfície Corrugada de $L_{0}$ variável, $L=3 \mathrm{~mm}$, $h=1 \mathrm{~mm}, \Delta \mathrm{P}=10 \mathrm{~Pa}, H=5 \mathrm{~mm}$.

2.6 Geometria para uma superfície corrugada de $L_{0}$ variável, $L=3 \mathrm{~mm}$, $h=1 \mathrm{~mm}, \Delta \mathrm{P}=10 \mathrm{~Pa}, H=10 \mathrm{~mm}$.

2.7 Geometria para uma superfície corrugada de $L_{0}=1 \mathrm{~mm}, L=3 \mathrm{~mm}$, $h=$ variável, $\Delta \mathrm{P}=10 \mathrm{~Pa}, H=$ variável.

3.1 comparação de $f \times R e$ para uma placa plana experimental e teórica

3.2 comparação de $f \times R e$ para uma placa plana e uma placa com ranhuras de $L o=0,3 \mathrm{~mm}$

3.3 Teste de longa duração 
Un abîme effrayant, une profusion de questions de toutes sortes où ma responsabilité était en jeu se présentaient à moi. Et la plus importante: qu'est-ce qui doit remplacer l'objet manquant? Le danger d'un art ornemental m'apparaissait clairement, la morte existence illusoire des formes stylisées ne pouvait que me rebuter.

C'est seulement après de nombreuses années d'un travail patient, d'une réflexion intense, d'essais nombreux et prudents où je développais toujours plus la capacité de vivre purement, abstraitement les formes picturales et de m'absorber toujours plus profondément dans ces profondeurs insondables, que j'arrivais à ces formes picturales avec lesquelles je travaille aujourd'hui et qui, comme je l'espère et le veux, se développeront bien plus encore.

Il a fallu beaucoup de temps avant que cette question: 'qu'est ce qui doit remplacer l'objet ?' trouve en moi une véritable réponse. Souvent je me retourne vers mon passé et je suis desespéré de voir combien de temps il m'a fallu pour arriver à cette solution.

Wassily Kandinsky, Regards sur le passé. 


\section{Sumário das notações}

\section{Símbolos Romanos}

A Comprimento da parede deslizante

$D_{h} \quad$ Diâmetro hidráulico

$f \quad$ Fator de atrito

$g \quad$ Função de distribuição nodal

$H \quad$ Altura do canal

$h \quad$ Altura da ranhura

$\overline{\bar{I}} \quad$ Tensor unitário

L Comprimento da geometria

$L_{0} \quad$ Comprimento da ranhura

$P \quad$ Pressão

$P^{*} \quad$ Pressão

$Q \quad$ Vazão de uma superfície com ranhura

$Q_{0} \quad$ Vazão de uma superfície sem ranhura

$R_{e} \quad$ Número de Reynolds

$s$ Comprimento de arco ao longo da linha

$\overline{\overline{T_{i}}} \quad$ Tensor das tensões

$\overrightarrow{v_{i}} \quad$ Vetor velocidade

$\vec{V}_{W} \quad$ Vetor velocidade na parede

\section{Símbolos Gregos}

$\rho_{i} \quad$ Densidade do líquido

$\mu_{1} \quad$ Viscosidade do líquido 1

$\mu_{2} \quad$ Viscosidade do líquido 2

$\beta$ Coeficiente de deslizamento

$\sigma$ Tensão da interface 